\title{
Repeated Sparks Produce Free Energy: A Rigorous Mathematical Explanation of the Authors, by the Hidden Ampere Force Law
}

\author{
Panos Pappas ${ }^{1}$, Theocharis Pappas², Eleuterios Pappas ${ }^{2}$ \\ ${ }^{1} 26$ Markopulioti Streer, Athens, Greece \\ ${ }^{2}$ Physics Department, Athens University, Athens, Greece \\ Email:ppappas@papimi.com,tpappas@papimi.gr,lpappas@papimi.gr
}

How to cite this paper: Pappas, P., Pappas, T. and Pappas, E. (2018) Repeated Sparks Produce Free Energy: A Rigorous Mathematical Explanation of the Authors, by the Hidden Ampere Force Law. Journal of Applied Mathematics and Physics, 6, 155-160. https://doi.org/10.4236/jamp.2018.61015

Received: November 14, 2017

Accepted: January 14, 2018

Published: January 17, 2018

Copyright $\odot 2018$ by authors and Scientific Research Publishing Inc. This work is licensed under the Creative Commons Attribution International License (CC BY 4.0).

http://creativecommons.org/licenses/by/4.0/

\begin{abstract}
This is the twinned paper of the increased mass paper, "Gravity is acting at superluminous infinite speed, thus collapsing special theory of relativity of Einstein, an explanation for almost everything (according to the authors' rigorous mathematical proof and overwhelming experimental evidence)" [1]. In the present paper, we describe the repeated spark or repeat current in terms of the hidden, Non-Relativistic Longitudinal Cardinal Ampere Force which is not found yet infallible for every case. The impressive finding is the fact that these repeated sparks as we shall see, have no back-emf, but forward-emf and forward-Ampere-motive-force. The only consumption is the resistive load with the very high currents needed. This means its total energy consumption is less than the released energy-output work plus heat, plus the energy recharging the batteries. All these are in excess of the consumed input energy. This means the Repeated sparking is "over-unity" efficiency and that, using super-conductive materials, we shall have constructed the first "perpetual mobile" plus producing extra electrical energy. Several previous journals that we did not mention here their names did not dare to publish our disliked papers of very important and revolutionary results but were not able to counter argue our arguments. They preferred the silence and have not answered us at all for many years now. Even some of them were involved previously with the repeated sparking which is the same topic of our present paper.
\end{abstract}

\section{Keywords}

Repeated Sparking, Ampere Force, Production of Energy, Perpetual Mobile, Spark, Lightning, Revolutionary Paper, Free Energy

\section{Introduction}

The repeated sparking is not a practical and common for the need of use of very 
high currents. However, it is a very interesting theoretically for having partially non-closed circuits due to the finite speed of the spark. For this reason, the usual Lorentz force law is inappropriate. The correct for this particular case, Ampere force law may be used only instead.

The Ampere force was hidden and was very little known and almost unknown in the literature for 180 years, since 1826, and until the Authors published [2] about it first in 2014.

The correct Lorentz force is only when it coincides with the closed integral of the Ampere force for absolutely closed electric current circuits, when the later force is integrated along the closed circuit which acts as the source of the "magnetic" field and then only coincides with the Lorentz force. In every other case, the correct Ampere force integral is not the same as the Lorentz force and this Lorentz force is incorrect, violating [3] momentum, angular momentum, causing self-propulsion, arbitrarily assumed to cause a strong an $\mathrm{E} / \mathrm{M}$ radiation to compensate conservation of momentum and angular momentum, experimentally the radiation [3] was never observed, but falsified. However, the Ampere force law may be used in all relevant cases with or without the exceptions of the Loretz force law. For example, the Faraday induction caused along a circuit is a longitudinal motive force along a circuit, compatible and predicted [3] by the Ampere force, and not compatible by the Relativity and contrary to the transverse to the conductor-current Lorentz force, at all.

The Ampere force alone makes the Ampere Electrodynamics, much simpler than Maxwell's Electromagnetism with the unsolvable Maxwell's equations. The Ampere Electrodynamics determines directly all the motive forces and Electromagnetic waves [2], with no equation solving, which together with the Coulomb law is all that is needed for Electromagnetism or better Electrodynamics.

\section{Explanation}

We performed a rigorous explanation of the "Repeated sparking" by the longitudinal non-relativistic Ampere force. The Ampere force also obeys correctly the action-reaction principle of Newtonian Mechanics that today is valid in everyday life. Never, and nowhere it has been falsified. On the contrary, the Lorentz force does not obey Newtonian mechanics, violating momentum, angular momentum principles of conservation, never observed true in practice [3] [4].

Here, we shall discuss the main dominant and fundamental principle of operation of the so-called repeated sparking, based on the longitudinal, non-relativistic, Ampere contact force:

$$
\mathrm{d}^{2} F_{12}=k \frac{r_{12} I_{1} I_{2}}{r_{12}^{3}}\left\{2 \mathrm{ds} s_{2} \cdot \mathrm{ds}_{1}-\frac{3}{r_{12}^{2}} \mathrm{~d} s_{2} \cdot r_{12} \mathrm{ds}_{1} \cdot r_{12}\right\}
$$

where $\mathrm{d} s_{1}$ is the infinitesimal length which contacts the current $I_{1}$ and produces the infinitesimal force $\mathrm{d} F_{12}$ on the infinitesimal section $\mathrm{d} s_{2}$, curing the current $I_{2}$ or 


$$
\mathrm{d}^{2} F_{12}=\left(\mu_{0} / 4 \pi\right) \mathrm{d} q_{1} \mathrm{~d} q_{2}\left(r_{12} / r_{12}^{3}\right)\left\{2 V_{1} \cdot V_{2}-\left(3 / r_{12}^{2}\right) V_{2} \cdot r_{12} V_{1} \cdot r_{12}\right\}
$$

taking into account $I \mathrm{~d} s=(\mathrm{d} q / \mathrm{d} t) \mathrm{d} s=\mathrm{d} q \mathrm{~d} s / \mathrm{d} t=\mathrm{d} q v$, and $\mathrm{d} s / \mathrm{d} t$ is the drift velocity $V$ of electrons [5], $\mathrm{d} q$ is the infinitesimal total charge $=n A q$.

Finally the Ampere force parametrized by the motive parameters of the drift velocities $V_{1}, V_{2}$ of the two charges $\mathrm{d} q_{1}, \mathrm{~d} q_{2}$ involved.

For $F_{12}=\lambda r_{12}, F_{21}=\lambda r_{21}, \lambda>0$, we have a repulsion, $F_{12}$ and $r_{12}, F_{21}$ and $r_{21}$ have the same direction. That means, the endpoints of a non-closed current straight line section of a spark, or the straight parts of a lightning repel each other generating energy for both the spark and the lighting. The latter case charges the Geo-electric field [6].

With similar $\mathrm{d} q$ and opposite $V s, \lambda<0$, we have an attraction.

For an isolated current like lightning falling from the sky, the endpoints of lightning repel and the lighting becomes self-propelled, gaining energy. Thus, lightning charges up, not discharges, the Geo-electric field by offering energy to it and thus maintaining it, according to meteorology ([6]: lightning).

$90 \%$ of lightning brings negative charges to the negative ground [6], instead of going upwards to the positive and more conductive ionosphere, if it was due to electrostatic attraction, assumed between the ground and a cloud. Moreover, this is impossible, because the lightweight and very flexible cloud should be attracted downwards. An electrostatic spark break is due to 1000 per mm. Lightning is usually several $\mathrm{Km}$ long [6]. So a cloud should have been more often negatively charged to several hundred billion volts exceeding the maximum of the positively charged ionosphere of 400,000 volts! On top, lightning appears between differently charged clouds or the different parts of the same cloud. This seems impossible because charges on a cloud are of similar origin and can't be of different sign.

Similar small charges on a cloud are pushed by the wind, causing a non-closed section current of moving charges. The currents might be in different directions, depending on the direction of the wind. Thus, lighting is self-created, self-propelled and self-energy producer and powered, breaking in many different directions, no evidence of particular potential difference direction. It does not follow a particular direction. It is self-propelled and is breaking in many different directions, even reversing direction by 90 or even 180, 360 degrees. Often the lightning extinguishes, terminating to nowhere. Many times, lightning trajectories cross one another without following any conservative field, such as the electrical field, for which classical theory proves its lines of field can not cross each other. For confirmation, see the pictures of lighting farther below: Lightning is not the first phenomenon of energy production. Energy production appears in many phenomena as sparks, lightning, similar to mass production [1] and particularly those associated with Ampere force law, as well as other force laws. Those who are interested may visit us to see and thoroughly examine our standing-by energy production apparatus for this purpose, consisting of an advanced digital oscilloscope, a square pulses generator, an electronic board for defining the 


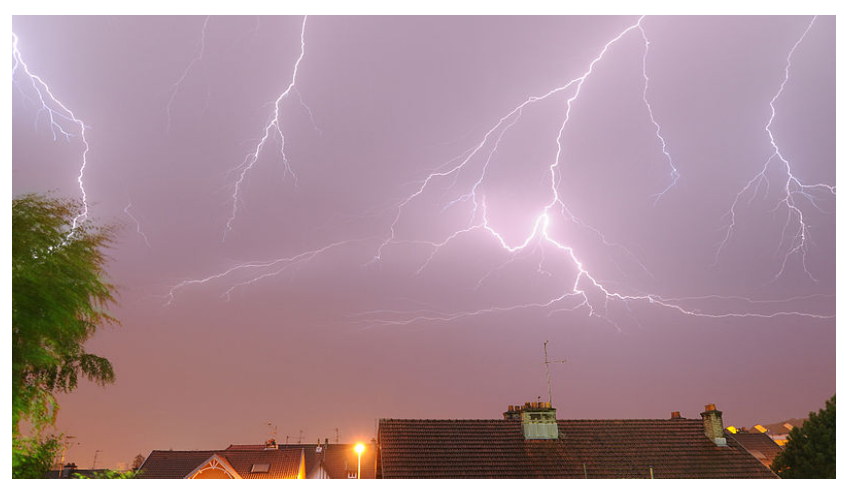

(a)

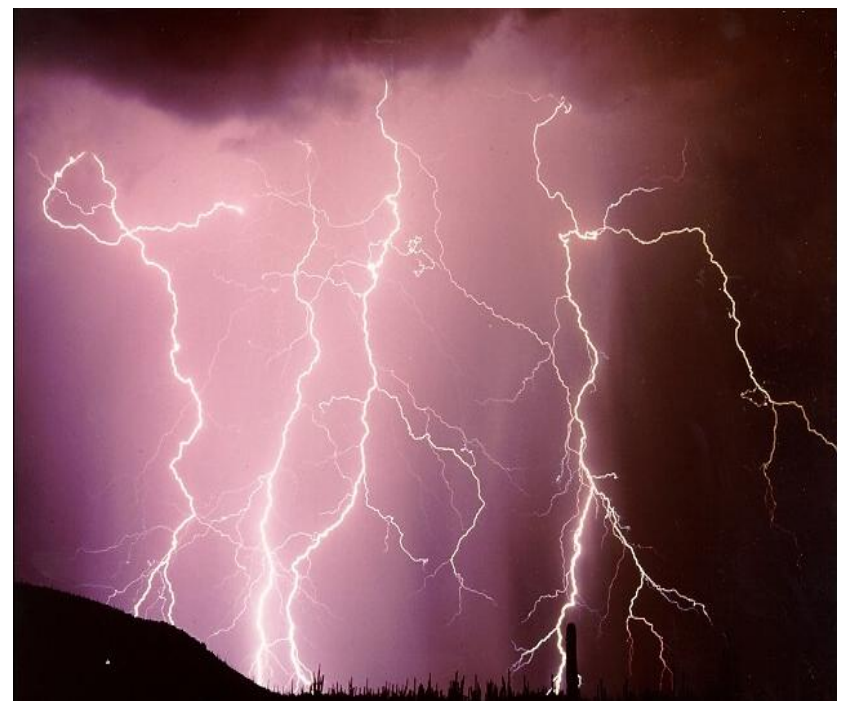

(c)

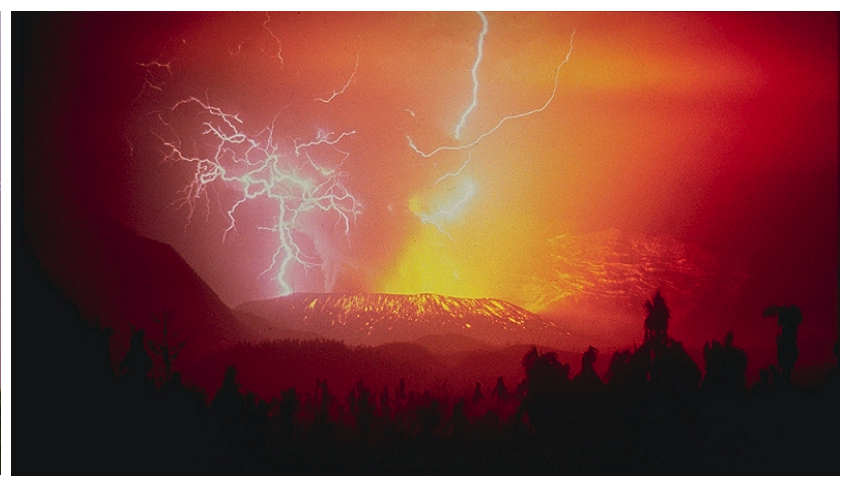

(b)

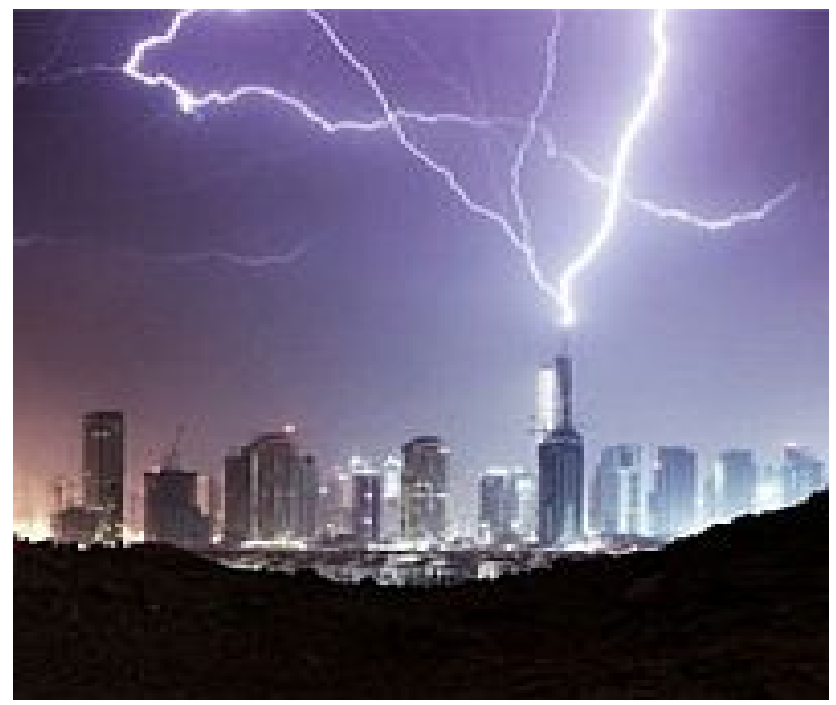

(d)

Figure 1. Lightning's self propulsion and self energy.

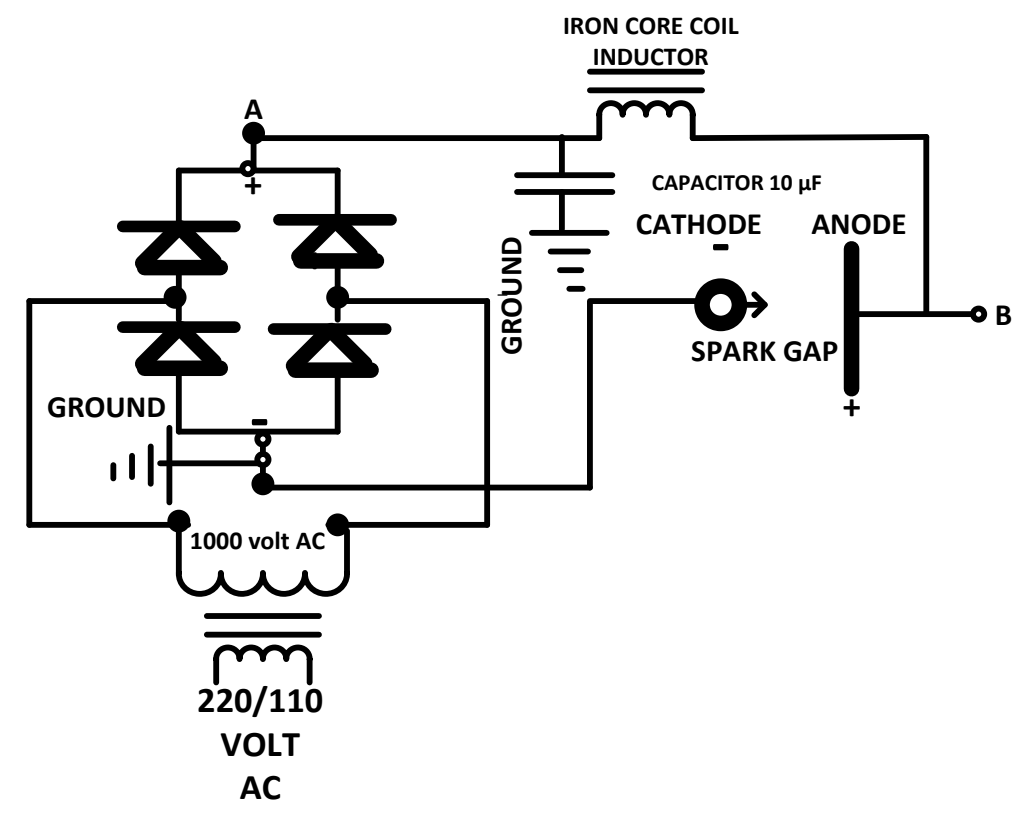

Figure 2. Free energy producer. 
direction of the pulses, identical light bulbs for realistic energy direct comparison, confirming the abstract, so to speak, oscilloscope images, in Athens Greece and welcomed to stay for as long they wish, eventually until they will be convinced, preferable physicists, knowing electronics or just electronic engineers! Contact us to make arrangements, our email is indicated under the title of the present.

From Figure 1, we have another experimental evidence for the Ampere force that is self-propelling lightning and offering excess energy to it.

Due to the fact an isolated spark, because of the finite speed of propagation electric current repels its ends and behaves like a compressed spring when it is released, point $B$ with respect to the ground, surpasses the potential of point $A$ with respect of the ground (Figure 2). This has clearly increased energy with respect to the ground.

\section{Conclusion}

For, the first time, the proper analysis of the repeated sparking is made. The basis was the only appropriate and infallible cardinal [7] force of Ampere. We have also proven here, for the Repeated sparking, it is an energy generation phenomenon and into a certain extent it is self propelled by well-established laws of Physics. The non-relativistic absolute Ampere force law is always applicable [7]. We note: the relativistic Lorentz force is only correct [8], when it coincides [8] with the integral of the absolute Ampere force. Here we have partial isolated-non closed current in the sparks. So, the integral of Amper force does not coincide with the Lorentz force. The Lorentz force is not correct when it does not coincide [8] with the Ampere, Equation (1), Equation (2), integral. So it is safe and wise to use always the absolute Ampere force, and risky [8] and unwise to use the usual Lorentz law; as in this case, the relativistic Lorentz force is an approximate law and is only to use carefully in special cases [8], but it is completely inappropriate for the present case, the general Ampere force law can unconditionally be used always in all cases.

\section{References}

[1] Pappas et al., Mass production; gravity is acting at superluminous infinite speed, thus collapsing Special Theory of Relativity of Einstein, an explanation for almost everything, (according to the authors' rigorous mathematical proof and overwhelming experimental evidence), submitted for publication to physical science international, sciencedo maininternational.

[2] Pappas, P.T., Pappas, L.P. and Pappas, T.P. (2014) Ampère Cardinal ForcesElectrodynamics-Proof and Prediction of Empirical Faraday Induction. Physics Essays, 27, 570-579.

[3] Pappas P. T., Violation of basic laws, "Induction in the dragged Dirac sea of particles-antiparticles", Producing the known e/m waves, with velocity c, with respect to it, SUBMITTED ON 11/15/2017. TO: Journal of Applied Physical Science International. http://resources.schoolscience.co.uk/cda/16plus/copelech2pg3.html

[4] Pappas, P.T. and Vaughan, T. (1990) Forces on a Stigma (Z) Antenna. Physics Essays, 3, 211. 
[5] Drift Velocity and Current.

http://resources.schoolscience.co.uk/cda/16plus/copelech2pg3.html

[6] Golde, R.H. (1977) Physics of Lightning. Academic Press, Vol. 1, p. 317.

[7] Maxwell, J.C. (1891) A Treatise on Electricity and Magnetism. Clarendon Press, Oxford, UK. ibid., unabridged third edition Dover Publications, Inc., New York 1954, "Infallible Cardinal Law of Ampere", Vol. 2, p. 175.

[8] Google Search: Proof of Equivalence of Ampere and Lorentz Law, for Example, Chritodoulidis Ampere Lorentz Equivalence.

http://www.physics.ntua.gr/ cchrist/-\%20C.\%20CHRISTODOULIDES/PERSONA L/PUBLICATIONS/Christodoulides\%2026.pdf 